\title{
La educación como garantía de igualdad o desigualdad en la sociedad moderna: Observaciones desde la teoría de sistemas sociales
} (2)

\author{
Education as a guaranty of equality or inequality in the modern \\ society: observations from social systems theory
}

Julio Labraña

Universidad Federico Santa María, Chile

\begin{abstract}
Resumen
La sociología de la educación es una de las especialidades más antiguas de la teoría sociológica. Derivado de las anotaciones de los clásicos, existe hoy consenso entre teorías funcionalistas, críticas y del capital humano en considerar a la educación como un ámbito desde el cual pueden extenderse relaciones de igualdad o desigualdad en la sociedad. El presente artículo pone en cuestión este supuesto en base a la disminución de la relación anteriormente asegurada entre educación, política y empleo. Proponemos frente a este fenómeno la utilización de la teoría de sistemas sociales pues ofrece una alternativa a partir de la introducción de los conceptos de diferenciación funcional, codificación autorreferencial y diferencia entre inclusión y exclusión. Posteriormente revisamos investigaciones en el sistema educativo basadas en estas premisas para finalmente concluir con indicaciones derivadas de lo expuesto.
\end{abstract}

\begin{abstract}
The sociology of education is one of the oldest specialties on the sociological theory. Derived from the classic's annotations, there is an agreement between functionalist and critical theorists, including human capital which considers the education as a field where the relations of equality and inequality could be spread in the society. This article questions the assumption basing on the decrease of the previous relation among education, politic and employment. The idea on this phenomenon is to use the society system theory because it offers an alternative in the introduction of the functional differentiation's concepts self- referential encoding and difference between inclusion and exclusion. Later on, it will be checking the investigations on the educational system based in these questions to conclude with indications derived of the investigation.
\end{abstract}

Palabras Clave: Sistema Educativo; Niklas Luhmann; Desigualdad; Inclusión; Exclusión Keywords: Educative System; Niklas Luhmann; Inequality; Inclusion; Exclusion

\section{Introducción}

El propósito de las siguientes reflexiones es exponer parte del panorama general de la sociología de la educación para plantear, en asociación con la teoría de sistemas del sociólogo alemán Niklas Luhmann, alternativas a la observación de la extensión de relaciones de igualdad y desigualdad a través de la educación sobre la sociedad. Dicho en otros términos, postulamos que tanto socialización como mérito en la educación presentan un estímulo para la educación, siendo tematizados en forma contextual. La 
intención del artículo es modesta: no es el objetivo realizar un recuento exhaustivo de las críticas ni tampoco una síntesis de los estudios empíricos sobre el tema desde la teoría de sistemas como ha sido realizado en otros trabajos (Massé 2007, Qvortrup 2005). Más bien, estas serán tomadas en cuenta únicamente como medios para proponer una nueva observación de la desigualdad en la educación y de esta forma incorporar irritaciones que puedan hacer necesarias la formulación de correcciones en la teoría de sistemas.

Para abordar este objetivo el artículo se inicia con "la unidad del debate por la educación" donde doy cuenta de las discusiones tradicionales en la sociología de la educación y los presupuestos compartidos que presentan perspectivas teóricas tecnocráticas, críticas y funcionalistas en su concepción del aparato educativo como espacio privilegiado para superar una desigualdad que la sociedad moderna ha dejado de aceptar (I). A continuación expondré las diferencias de este paradigma respecto a la educación en la teoría de sistemas en el marco de la diferenciación funcional bajo el título "El sistema de la educación como un sistema social" (II). Posteriormente, en "La utilización de la forma inclusión/exclusión en el sistema de la educación" (III) compararé cuatro formulaciones teóricas que permiten abandonar la idea de la educación como espacio privilegiado para superar la desigualdad respetando la autonomía del sistema educativo (Ossandon, Robles, Mascareño y Michailakis \& Reich), para finalmente concluir con una breve reflexión sobre lo anteriormente expuesto (IV).

\section{La unidad del debate por la educación}

La preocupación por la educación tiene una larga data en la sociología. Ya en los escritos de los primeros teóricos sociales como Saint-Simon y Comte queda de manifiesto un planteamiento sociológico sobre las funciones que le corresponde cumplir a la educación para asegurar el progreso de la sociedad moderna. Los cambios sociales de la época expresados en la transición desde la sociedad aristocrática del antiguo régimen a la sociedad industrial fueron percibidos como una pérdida de los fundamentos sociales naturales, hecho que quedó de manifiesto en el aumento de la tasa de suicidios, pérdida de legitimidad del Estado, crisis económicas, ausencia de valores comunes, entre otros. Este fenómeno de pérdida del vínculo social hizo que progresivamente las desigualdades naturales dejasen de ser legítimas por la adscripción a un estrato. Ni siquiera la apelación a una cosmogonía religiosa puede ya asegurar la integración social (Luhmann 1998).

Derivado de este problema, la sociología de la educación desde sus inicios se fue constituyendo como una sociología de las instituciones escolares marcando con énfasis funciones orientadas a la integración, el control social y la asignación de roles por variables adquiridas y no adscritas como el lugar de nacimiento. El primer libro clásico sobre el tema, "Educación y sociología" de Durkheim rechaza las pedagogías idealistas de Kant, Herbart y Pestalozzi para destacar que la educación no es un ideal alejado de variables históricas sino es el resultado de las necesidades que la sociedad tiene en una determinada época. En el caso de las sociedades europeas, los problemas corresponden al debilitamiento de los vínculos sociales provocados por la división del trabajo y la consiguiente pérdida de procesos de interiorización de moralidad (Nisbet 1974).

En este sentido, justamente debido a sus características de obligatoriedad e intencionalidad específica, la escuela permite frenar las tendencias anómicas mediante la homogeneización de una serie de estados físicos, intelectuales y morales en la población. Como señala Durkheim, tanto burgueses como obreros viven en un medio idéntico, son miembros de una misma sociedad $y$, por consiguiente, no pueden dejar de estar impregnados por idénticas ideas (Durkheim 1991: 65). De esta forma, la función de la educación está en desarrollar aptitudes generales hacia la moralidad en el alumno de modo que se convierta en alguien capaz de vivir en sociedad. Sólo de esta forma la educación asegura que la desigualdad sea "proporcional al mérito de cada uno" (Durkheim 1969: 238), transformándose en la punta de lanza de una sociedad en que el origen no 
sea relevante.

A partir de las transformaciones industriales ocurridas durante el siglo XX las teorías funcionalistas profundizaron sus planteamientos en la función de la escuela como garante simultáneo de unidad moral y diferenciación laboral. Con un hincapié especial, la educación se convirtió en un objeto de estudio relevante tras la segunda guerra mundial al trasladarse la competencia por la hegemonía militar entre potencias desde el ámbito económico al de la producción tecnológica (Bonal 1998:30). En este contexto la reflexión de Parsons propone un mayor desarrollo teórico sobre las funciones de la educación. Para el sociólogo norteamericano el problema que la educación debe satisfacer en la sociedad moderna es la necesidad de internalizar compromisos normativos a la vez de promover la asignación de recursos para la estructura de roles o, según él mismo, "conservar los valores más fundamentales de la sociedad" a la vez que se posicione el "desempeño de un tipo específico de rol dentro de la estructura social" (Parsons 1959).

Combinando la lectura de Durkheim con los esquemas de Weber orientados a la acción, Parsons postula que en las sociedades modernas existe una clara tendencia a la producción de desigualdad producto únicamente de los logros académicos derivados de la capacidad propia de cada estudiante y no sobre factores como el sexo o el estatus socioeconómico. Si bien el sociólogo estadounidense reconoce el peso de estas variables en la sociedad norteamericana asume su disminución constante en el tiempo pues "el principal proceso de diferenciación (que, desde otro punto de vista, es la selección que se efectúa en la escuela primaria) gira en torno a un solo eje: el desempeño" (Parsons 1959: 3). En este sentido, el sistema educativo moderno, único con estas características, estaría en una posición privilegiada para asegurar la integración a la vez que instaura la primacía del mérito en la asignación de roles en la estructura de la sociedad. Dicho en otros términos, la educación forma de la promoción del consenso de valores que reacciona a la diferenciación de roles con una generalización también creciente.

Esto queda de manifiesto cuando observamos que la escuela hace posible la emancipación del niño de la influencia restringida de la familia mediante la asignación en los primeros años de un profesor único, largas jornadas escolares y relaciones con pares no familiares. Utilizando la terminología parsoniana, esto facilita el reemplazo de variables pauta particularistas a unas orientadas por criterios universalistas con lo que se aseguran las expectativas normativas mínimas que la convivencia en la sociedad requiere. Por otra parte, puesto que la escuela únicamente diferencia según el desempeño en evaluaciones y exámenes, la diferenciación social mitiga las tensiones que ella misma genera. (Parsons 1959). A partir de estas características, la perspectiva de Parsons asume la continuidad entre las escuelas y el resto de la sociedad en la regulación de los problemas provocados por la diferenciación de la sociedad. El universalismo impuesto en la sala de clases por los profesores asegura la integración social así como la primacía del mérito afirma la diferenciación en roles complementario en el resto de sistemas de la sociedad.

Particularmente desde la década de los setenta la eficacia de las políticas educativas en torno a la promoción de una desigualdad acorde a factores adquiridos fue puesta en cuestión por una serie de movimientos sociales que, retomando la teoría marxista, criticaron el fracaso de la educación como mecanismo de distribución social. Esta situación condujo a los gobiernos europeos y al gobierno estadounidense a investigar el fenómeno, adjudicando causalidades a la falta de recursos económicos destinados a la educación. Sin embargo, contrario a estos supuestos el Informe Coleman (1966) indicó que la explicación de la correspondencia entre clase social y resultados académicos estaba en la privación cultural de las familias y no en el tipo de escuelas al que asistían. Tanto este como otros estudios que continuaron investigando esta tesis resultaron notablemente problemáticos para las premisas de las teorías funcionalistas. La investigación de Jecks, "Inequality" (1972), señaló la desconexión entre posición educativa (desempeño), estatus ocupacional e ingresos para desacreditar los presupuestos funcionalistas de relación entre 
socialización, educación e integración en el mundo laboral. En idéntica dirección Thurow (1983) hizo notar que pese a haber ascendido en los países del tercer mundo el nivel educativo de la población entre 1950 y 1970, la pobreza no había dejado de incrementarse. De esta forma quedaba puesta en entredicho la función de diferenciación laboral asociada al mérito académico del estudiante en las instituciones escolares.

Sin embargo, pese a poner en cuestión los supuestos funcionalistas de idéntica accesibilidad a los contenidos educativos, diferenciación producto de criterios académicos y la conexión entre resultados escolares y posición en la estructura laboral, los estudios anteriores mantuvieron intacta la concepción de un sistema educativo que, si bien imperfecto, era neutral (Bonal 1998). Las teorías críticas, en cambio, se presentaron a sí mismas como desveladoras de la función de la educación para mantener las diferencias entre clases sociales antagónicas. Para estas teorías la desigualdad no es consecuencia de una imperfección en el funcionamiento del aparato escolar sino más bien el rol que le corresponde cumplir a la educación en determinada etapa de desarrollo del capitalismo.

La idea central de los textos de Bourdieu sobre la educación, por ejemplo, es que la arbitrariedad en la selección de determinadas formas de cultura favorece sistemáticamente a los individuos provenientes de familias con un mayor capital cultural y segrega a aquellos de menores recursos que carecen de este. En 1964 el autor publica junto a Passeron la investigación "Los estudiantes y la cultura", donde señala que las principales limitaciones para los estudiantes de bajos ingresos de la Universidad de Paris no son de tipo económico, como podría argumentar el funcionalismo, sino culturales pues las evaluaciones presuponen capacidades cuya adquisición en familias de bajo capital cultural es improbable (Bourdieu \& Passeron 2003: 45). En efecto, en los exámenes los profesores universitarios tenderían a valorar la creatividad y la asociación abstracta por sobre la aplicación empírica de conceptos. Dado que los alumnos provenientes de familias de menores recursos han sido socializados para orientarse a aspectos prácticos y no intelectuales esta desventaja social se realiza como un fracaso del propio alumno, en tanto el desempeño superior de alumnos provenientes de familias de mayores ingresos no se observa como consecuencia del contexto familiar sino de dones y talentos individuales.

Estos criterios de evaluación que favorecen a las familias de clases altas no se derivan de una jerarquía de conocimientos sino que son producto de luchas de poder en el seno de la estructura social de modo que finalizan otorgando legitimación a una clase sobre otra. Como señala el sociólogo francés, "por el hecho de que corresponden a los intereses materiales y simbólicos de grupos o clases distintamente situados en las relaciones de fuerza, estas AP (acciones pedagógicas) tienden siempre a reproducir la estructura de la distribución del capital humano entre esos grupos o clases, contribuyendo con ello a la reproducción de la estructura social" (Bourdieu \& Passeron 1977: 51). De esta forma la supuesta autonomía con la cual la educación asegura su independencia respecto a variables de nacimiento es sólo el modo cómo se oculta que es en la escuela y la universidad donde la diferencia económica entre grupos se legitima mediante la asociación entre capital cultural y logro académico como criterio de diferenciación entre los alumnos.

Donde Bourdieu establece la arbitrariedad de los criterios educativos, Bernstein se interroga sobre los mecanismos específicos que favorecen y desfavorecen a ciertas clases sociales. Debido a que la posición de las familias en la división del trabajo determina el lenguaje que estas utilizan, la institucionalización en el aparato educativo de cierto código lingüístico termina por naturalizar determinadas formas de desigualdad. Para el sociólogo británico existen dos estructuras lingüísticas opuestas: el código restringido y el código elaborado o formal. Mientras en el primero, utilizado por las clases bajas, priman expresiones gramaticales cortas fuertemente dependientes de las experiencias vitales, en el segundo, característico de las clases medias y altas, destacan construcciones gramaticales complejas, la utilización de pronombres impersonales y el desarrollo de una 
mayor conceptualización (Bernstein 1985: 45). Dicho en otros términos, el código restringido está diseñado para la descripción de experiencias concretas en tanto el código elaborado hace probable la elaboración de abstracciones de una complejidad mayor.

Dado que el sistema educativo ha evolucionado con pretensiones universalistas y de abstracción es natural que el código elaborado rija el currículo, la pedagogía y las formas de evaluación dejando automáticamente en desventaja a quienes hubiesen sido socializados en un código lingüístico más restringido. Por otra parte, debido a la importancia de la educación en la sociedad actual, las instituciones escolares y su selección del código elaborado promueven que desigualdades de cuna se mantengan gracias al (y no a pesar del) aparato escolar. Como menciona Bernstein, "cómo una sociedad selecciona, distribuye, transmite y evalúa el conocimiento educativo, que ella considera debe ser público, refleja tanto la distribución del poder como los principios del control social" (1985: 13).

Desde una perspectiva distinta pero conservando el análisis crítico de la educación, Althusser señala las funciones de la educación en la sociedad capitalista a partir de la introducción del concepto de aparatos ideológicos del Estado. Junto con la Iglesia y la familia, la escuela se presentaría como uno de los agentes donde imponer la ideología burguesa en el conjunto de la sociedad y legitimar como naturales las desigualdades entre clases sociales. Dado que está menos subordinada a las relaciones con el Estado, la educación se presentaría como "relativamente autónoma" ofreciendo un aspecto menos conflictivo para la expresión de las contradicciones propias de la lucha de clases capitalista y la lucha de clases proletaria (Althusser 1985: 308). No por esto debe entenderse que la educación es un aspecto secundario en la naturalización de las relaciones de poder. Precisamente por el importante rol que cumple en la actualización ideológica de las conciencias, la transformación de ésta sin mediar cambios en la estructura de los medios de producción es imposible. De esta forma, el sistema de la educación realiza funciones de socialización que transforman en naturales desigualdades que expresan las contradicciones de las esferas de producción capitalistas.

La tesis de Bowles \& Gintis se basó inicialmente en la descripción de la correspondencia existente entre la estructura económica y la superestructura ideológica en el ámbito de la educación. Los autores señalan la presencia de un contínuum entre las relaciones escolares y las de la producción, no en torno al mérito, sino respecto a las jerarquías, grados de injerencia, control o productividad. En este sentido, la relación entre profesor y alumno actuaría como una metáfora de la relación entre el dueño de los medios de producción y el trabajador así como la participación del alumnado en los planes de estudio sería tan vacía como la del trabajador en los planes futuros de desarrollo en la empresa (Bowles y Gintis 1976:175).

Esta descripción permite a los autores asignar al sistema educativo la función de constituir las formas de personalidad necesarias para la estructura productiva capitalista. Los individuos de clases inferiores aprenden en la escuela a obedecer normas pasivamente en tanto los hijos de clases de mayores recursos desarrollan una mayor independencia y autonomía para emprender. Como señalan Bowles \& Gintis, "las relaciones jerárquicas están reflejadas en las líneas de autoridad verticales que van de administradores a maestros y de maestros a estudiantes. El trabajo enajenado se refleja en la falta de control que tiene el estudiante sobre la educación, la enajenación de éste sobre el contenido de sus planes de estudio y la motivación escolar a través del sistema de recompensas externas en lugar de mediante la integración del estudiante" (Bowles \& Gintis 1976: 175). De esta forma, la escuela se comprende como el espacio donde se socializa a las clases sociales hacia las normas requeridas por la estructura capitalista.

Conviven junto a las propuestas funcionalista y crítica una visión tecnocrática crecientemente dominante desde las reformas neoliberales, marcada por la recolección de 
datos económicos y promoción de políticas públicas con el máximo exponente de la teorías del capital humano. Los supuestos de esta teoría obedecen a una actualización del ideario funcionalista tras las variaciones en la estructura productiva al sector servicios, incorporando una concepción de la educación entendida como una inversión con tasas específicas de rentabilidad a nivel social (Bonal 1998:58).

Desde esta vertiente teórica las investigaciones han partido de la observación de los aparatos educativos como el espacio ideal para intervenir la desigualdad en la sociedad. Basados en este supuesto se elaboraron una serie de políticas públicas, especialmente en América Latina, en base a los consejos de organismos internacionales como la OCDE, UNESCO y el Banco Mundial. En Chile, por ejemplo, durante 1979 la Directiva de Educación definida por el Gobierno Militar señalaba que el Estado centraría sus esfuerzos en la educación básica para así capacitar "buenos trabajadores, buenos ciudadanos y buenos patriotas." Tras el retorno a la democracia en este país, la Comisión Brunner continuó con estas políticas al definir la educación como el redundo donde la "vida civilizada en comunidad" se hacía posible así como el logro de la democracia y la erradicación de la pobreza al hacer utilizables los talentos disponibles en provecho del país. Como señala el economista Harald Beyer, los beneficios de una buena educación se concentran en "contribuir a la competitividad y al crecimiento económico (...) elevando la productividad de nuestra fuerza de trabajo y potenciando el desarrollo de áreas de mayor valor agregado". Una buena educación permite construir una "sociedad de mejores personas y puede ayudar a reducir la desigualdad económica" del país (Beyer s/f: 2). De esta forma, según los seguidores de las teorías del capital, la educación permite desarrollar la moral social, el patriotismo, el crecimiento económico y la democracia.

Puede parecer este un panorama sumamente rico para la reflexión sociológica en tanto posibilita la convivencia de tres vertientes aparentemente opuestas entre sí. Mientras las perspectivas funcionalistas privilegiaban la observación de la unidad moral y diferenciación laboral según el mérito académico, la visión crítica destaca cómo mediante estos mismos mecanismos se asegura la reproducción de una sociedad estratificada en clases antagónicas entre sí. La opción tecnocrática decanta en el desarrollo de políticas públicas basadas en análisis económicos, orientados al bienestar general de la sociedad mediante una mayor cantidad de recursos asociados a las organizaciones educativas.

Sin embargo, y esta es mi tesis, en todas las aproximaciones es posible encontrar un núcleo sociológico común que considera como válida la posibilidad de extender las relaciones presentes en las salas de clases hacia el resto de la sociedad. En efecto, en las variantes funcionalistas la socialización promovida preferentemente en las escuelas constituye el "ser moral" de Durkheim y una "orientación universalista" en Parsons que permiten conservar las pautas normativas básicas que aseguran la integración de la sociedad. En el caso de las vertientes críticas, la educación o, más precisamente, la imposición de un modelo específico de educación, permite extender las desigualdades entre clases sociales como diferencias académicas y legítimas de rendimiento. Es en las escuelas donde se lleva a cabo la socialización, que un modelo antagónico de clases requiere para su supervivencia. Finalmente, en las concepciones derivadas del capital humano una mejora en las condiciones de educación incrementa las posibilidades de alcanzar una serie de beneficios en otros ámbitos de la sociedad sean estos morales, políticos o económicos. De esta forma, en todas las teorías mencionadas las relaciones presentes en las escuelas se extienden al funcionamiento de otros ámbitos de la sociedad. Sea equidad o inequidad, igualdad o desigualdad, la educación produce efectos lineales en el resto de la sociedad según el funcionamiento definido por cada teoría.

Considero posible proponer una interpretación que incorpore estos fenómenos y ofrezca una alternativa a esta unidad teórica, a partir de los lineamientos de la teoría de sistemas sociales del sociólogo alemán Niklas Luhmann, especialmente en el concepto de diferenciación funcional como característica central de la sociedad moderna. Enunciada en 
términos generales, la tesis es que la socialización en las organizaciones educativas no puede ser conceptualmente supuesta como extendida a otros sistemas de la sociedad. Esto significa que ni igualdad ni desigualdad pueden asegurarse a través de la educación.

\section{El sistema de la educación como un sistema social.}

La idea de que las esferas de la sociedad reflejan el todo mayor al cual pertenecen es el presupuesto del cual parten las citadas observaciones sobre la educación. Una escuela inmersa en una sociedad definida como de clases refleja estos antagonismos ya sea en la forma de evaluación (Bourdieu), lenguaje (Bernstein), falsa conciencia (Althusser) o construcción de personalidad (Bowles y Gintis). Desde la perspectiva del funcionalismo, la división del trabajo en base a variables adquiridas e integración de la sociedad se observa en la sala de clases tanto en la socialización del ideal moral (Durkheim) y orientaciones universalistas (Parsons) como en la diferenciación según méritos académicos. Del mismo modo, en una sociedad que aspira a la igualdad de oportunidades como la definida desde las teorías tecnocráticas, la educación tiende a promover estos ideales democráticos.

Contrario a ello, la teoría de sistemas sociales propuesta por Niklas Luhmann se basa en la comprensión de la sociedad como el total de comunicaciones que posibilitan la emergencia de sistemas operativamente cerrados, autorreferenciales y autopoiéticos encargados del cumplimiento de una función específica. Dicho en otros términos, toda irritación es procesada diferencialmente en cada sistema de la sociedad y no pueden ser extensibles de un caso a otro. En la sociedad contemporánea uno de estos sistemas es la educación. Comenzaré esta sección con una descripción de la teoría de la diferenciación sistémica para después dar cuenta de las especificidades de este sistema.

La diferenciación de la sociedad es el modo mediante el cual la propia sociedad resuelve sus problemas autocreados a través de la reintroducción de la diferencia entre sistema y entorno en el interior del sistema (Luhmann 1998:78). Luhmann distingue tres formas de combinación de la asimetría entre sistema y entorno frente a la diferencia entre igualdad y desigualdad: segmentación, estratificación y diferenciación funcional. Dado que la preocupación del presente trabajo es formular una alternativa a la observación de que los efectos de la educación se reproducen linealmente en otros sistemas, me concentraré en el último desarrollo evolutivo. En esta, la sociedad se organiza en subsistemas autónomos, clausurados operativamente y con un medio de operaciones único asociado a su función específica. Este es el caso de la verdad en la ciencia, el amor en las relaciones de intimidad, el dinero en la economía o el poder en la política. La autopoiesis del sistema queda determinada en el hecho de que cada sistema crea los elementos que le permiten mantener su unidad frente al entorno. Debido a que toda función debe ser satisfecha con idéntica premura en base a la interdependencia de los subsistemas, la sociedad no puede conceder primacía a ninguna, resultando por tanto una sociedad carente de centro único Como señala Luhmann, la diferenciación de la sociedad provoca que cada sistema observe las irritaciones en sus propios términos con lo que "nunca se trata del reflejo de distinciones que están previamente en el entorno" (Luhmann 2007: 88).

Estas consideraciones nos alejan ya de las observaciones de la educación como un campo cuyos efectos se dejan sentir linealmente en el resto de ámbitos de la sociedad. En una sociedad policéntrica como la funcionalmente diferenciada los sistemas sociales no pueden aspirar a coordinar sus operaciones según los criterios de otros sistemas. En la educación el rendimiento académico no puede asegurarse por el hecho de ser pobre o rico económicamente. Los sistemas funcionales son desiguales entre sí en torno a su función (la política es diferente del arte y de la educación), si bien presuponen una igualdad en torno a la exclusión únicamente según la función de cada sistema. Esto significa que la desigualdad se estructura únicamente como producto de las operaciones del sistema en cuestión (la economía tematiza sólo economía, la educación tematiza sólo educación), fenómeno con lo cual la estratificación pierde primacía como criterio de diferenciación. 
Por consiguiente, según Luhmann, puede observarse una tendencia a que los sistemas se vuelvan negligentes respecto a variaciones que no se correspondan con el medio de operación del sistema. En los intercambios económicos no son determinantes ni la moral ni las participaciones políticas así como tampoco el sufrimiento personal de cada uno de los participantes altera los efectos de la transacción (Luhmann 1994).

El sistema de la educación en la sociedad moderna lleva a cabo dos funciones aparentemente contradictorias: educación orientada al desarrollo de competencias y la observación de estas diferencias mediante la selección académica (Luhmann \& Schorr 1990). Mientras la comunicación educativa opera según el código de plausibilidad posible de enseñar/imposible de enseñar, la selección lo hace mediante la diferencia mejor/peor que, si bien puede variar en su programación, siempre distingue entre resultados aceptables y no aceptables, aprobados y reprobados, promovidos y no promovidos, y así sucesivamente. El acoplamiento de ambos códigos permite medir selectivamente en el sistema educativo si el aprendizaje se logró o no. En Chile, por ejemplo, de un alumno que obtiene una nota inferior a 4.0 en la enseñanza secundaria se presupone que no logró aprender lo académicamente relevante. Unos alumnos pasan de curso y otros no, unos son elogiados y otros castigados y esta diferenciación basada en el código de selección pedagógica permite atribuir posiciones en el sistema educativo.

Consecuentemente con el grado de diferenciación del sistema de la educación, la asignación de diferencias de rendimientos no puede articularse según valores trascendentales como los ideales de perfección o moralización o, los orientaciones más referidas actualmente, de modernización y desarrollo. En la diferenciación funcional no existen posiciones privilegiadas desde la cual promover efectos en el resto de sistemas, no hay cumbre, centro coordinador ni un mundo de "perfecciones absolutas, axiomáticas e inmutables" (Luhmann \& Schorr 1990: 56). En este sentido, Luhmann \& Schorr apuntan a que, por encima de promover efectos buscados en otros sistemas funcionales, el código de la educación actúa en forma negligente respecto a comunicaciones que no se correspondan con su medio. La sociedad funcionalmente diferenciada aspira a que la evaluación sea una e idéntica para todos: razón por la cual la determinación de desigualdades económicas resulta chocante en la actual sociedad.

Específicamente, el tipo particular de comunicación que maneja el sistema de la educación se realiza en la distinción entre intención de educar y la persona que se educa, el niño. Como señalan Luhmann \& Schorr, "a la intención de la educación corresponde la idea que el niño aprende o no aprende lo que debe aprender" (1996: 146). Esto le bastaría al sistema educativo para autoconfirmarse como un sistema autorreferencial: la intención educativa presupone un niño capaz de variar psíquicamente y este asume que sería algo diferente de lo que sería de no haber pasado por el sistema educativo.

De esta forma, antes que promover alteraciones en el resto de sistemas de la sociedad, el sistema educativo se orientaría a comunicar transformaciones en el entorno de la sociedad (sistema psíquico), fenómeno imposible de asegurar en la medida que sistemas sociales y psíquicos están clausurados uno frente al otro. Frente a esta situación, el sistema de la educación tematiza su propia comunicación con mayor comunicación; introduce herramientas como evaluaciones y exámenes que permiten aplicar el código de selección pedagógica y posicionar diferencias educativas a partir de diferencias educativas. En consecuencia, en la teoría de sistemas la educación promueve la individualización de los alumnos como alumnos (y nada más) en una mejor o peor trayectoria escolar de acuerdo a su desempeño medido autorreferencialmente.

Por tanto, mediante evaluaciones, elogios y promociones a nuevos cursos el sistema de la educación formula sus propias diferencias sin que estas puedan extenderse causalmente a otros sistemas. Si bien estas evaluaciones pueden tener un correlato en otros sistemas funcionales como el científico o el económico, adquieren su unidad propia en el interior 
del sistema. Como ejemplifica Luhmann, la escuela no maneja la diferencia entre seno y coseno sino entre los alumnos que saben manejar esta diferencia y los que no (Luhmann \& Schorr 1996: 46). Producto de la diferenciación funcional, para Luhmann, la aplicación del código de selección pedagógica produce únicamente diferencias relevantes para el sistema educativo. La indiferencia frente a las comunicaciones de otros sistemas tiene como consecuencia que alumnos con estudios de posgrado no tengan asegurado por esta característica la inclusión en el resto de sistemas funcionales de la sociedad.

Como puede seguirse hasta acá, el planteamiento expuesto comienza la reflexión teórica desde supuestos que ponen en cuestión la capacidad de extender los efectos de la educación hacia otros sistemas funcionales de la sociedad. La diferenciación funcional de sistemas que, si bien interdependientes, operan autorreferencialmente imposibilita considerar fenómenos que sean interpretados en forma idéntica entre sistemas. Una reprobación en el sistema escolar da lugar a que el alumno sea increpado por su familia (o no), ser definida como una característica judicialmente relevante (o no) o asegurar que por eso el alumno es una mala persona (o no). En los casos que acabamos de mencionar son los sistemas de la familia y el derecho junto al código de la moral, respectivamente, los que definen cómo los efectos de la educación se dejan sentir en cada sistema de la sociedad.

Esta teorización nos permite dar un paso adelante en el abordaje de la desigualdad en el sistema educativo entendiéndola, no como la correspondencia entre el funcionamiento de la educación y las posiciones asignadas en la sociedad, sino como el resultado del operar autorreferencial de los sistemas funcionales. En el mismo sentido, la educación promueve su indiferencia tematizando las operaciones de otros sistemas sólo en forma autorreferencial, esto es, educativamente. Las diferencias económicas entre los estudiantes son observadas en la educación como diferencias de rendimiento, así como tampoco se habla de Espíritu Santo sino de si el alumno tiene o no tiene talento. De esta forma, podemos observar dos formas de desigualdad educativa con características diferentes respecto a las definidas en las teorías sociológicas revisadas. Una desigualdad interna, resultado de la asignación autorreferencial de posiciones en el sistema educativo a través de la aplicación del código de selección pedagógica, y una desigualdad externa, que es la apropiación en otros sistemas sociales de la primera forma de desigualdad.

Esta última característica implica que las interrelaciones entre sistemas dejan de ser leídas como relaciones de causalidad para incorporarse en forma autorreferencial según el sistema afectado (Mascareño 2005). Paradójicamente, si bien la educación no puede asegurar cambios económicos ni políticos sí puede observarse económica y políticamente. Dicho en términos sistémicos, la diferenciación funcional promueve la autorreferencialidad en el operar de los sistemas sociales, fenómeno que transforman en improbables relaciones de causalidad entre la educación y el resto de la sociedad.

En la siguiente sección comenzaremos definiendo el concepto de desigualdad a partir del esquema de inclusión y exclusión, para posteriormente señalar autores que han analizado esta problemática distanciándose de la idea de correlación entre sistemas sociales. El criterio con el cual fue definida esta selección corresponde a que en estos autores se problematiza la suficiencia de la inclusión en una organización educativa para ser seleccionado pedagógicamente. Así, veremos cómo se pueden observar desigualdades internas y externas en el sistema educativo en la primacía de la diferenciación funcional.

\section{La utilización de la forma inclusión/exclusión en el sistema escolar}

Como ha destacado Massé (2007) parece ser poco productivo describir la desigualdad a través del concepto de exclusión entendida en el sentido tradicional sociológico del término como la incapacidad de acceder a determinadas organizaciones. El sistema 
educativo presenta dos problemas en este ámbito: por una parte una marcada tendencia desarrollada hacia la inclusión universal y, por otra, la convivencia de formas de inclusión más y menos rígidas. En América Latina, a partir de la década de los cincuenta, la educación primaria de América Latina y el Caribe se expandió hasta alcanzar, a fines de los ochenta, niveles prácticamente universales. En la educación secundaria la tasa bruta de ingreso se elevó de apenas un 15\% en 1960 a 54\% en 1992 (Caillods \& Maldonado 1997). De acuerdo a este fenómeno surgen visiones que afirman que la exclusión se relega a los niveles superiores del sistema educativo como es el caso de la enseñanza universitaria (MINEDUC 2008). Una segunda alternativa la presentan estudios que señalan que la desigualdad se encuentra en las diferencias entre organizaciones que median la inclusión educativa (Oviedo 1991; Contreras, Bustos \& Sepúlveda 2007). En el caso de Chile, las pruebas del Sistema de Medición de la Calidad de la Enseñanza (SIMCE) y la Prueba de Selección Universitaria (PSU) demuestran que las escuelas municipales dependientes del Estado tienen menores resultados a las escuelas privadas.

La formulación sistémica se integra a este debate a partir de la definición de la autorreferencialidad de los sistemas funcionales y la conceptualización de la diferencia entre inclusión y exclusión. A diferencia de la teoría parsoniana en que la igualdad social está asegurada por la integración en esquemas complementarios. De forma extremadamente simple, en este paradigma la sociedad está integrada en tanto todo individuo accede a un rol. Los que no son médicos son pacientes, quienes no compran venden y quienes no enseñan aprenden: en cualquiera de estos casos el sujeto es aceptado con un status más o menos completo de miembro de un sistema solidario mayor. El problema que Luhmann critica sobre Parsons es que este no responde qué ocurre cuando la integración no tiene lugar, fenómeno ante el cual propone, utilizando laxamente el cálculo de la forma propuesto por Spencer-Brown (1979), observar la inclusión como parte de una forma con una cara externa representada en la exclusión. A continuación explicaremos brevemente el efecto de estas conceptualizaciones para una teoría que señala la autorreferencialidad en el operar de los sistemas funcionales.

En la teoría sistémica, inclusión y exclusión actúan como un supercódigo sobre todos los sistemas de la sociedad. En términos prácticos, esto implica que no está excluido quién no pague sino de aquel que no se espere compra o venta alguna o, en el caso de la educación, no es excluido quién obtenga una mala calificación o repita un grado sino el alumno del cual no se espere la producción de nada académicamente relevante. Una segunda característica de la diferencia entre inclusión y exclusión consiste en la imposibilidad de obtener una regulación uniforme de la inclusión. Dado que la ordenación de las operaciones es realizada por cada sistema, la acumulación de efectos de inclusión es un fenómeno altamente improbable. Como señala Luhmann (1998), nadie pierde su capacidad jurídica por casarse sino lo más que puede perder en esta aventura es la fortuna. El fenómeno opuesto define a la exclusión dado que esta se integra de forma mucho más fuerte. El abandono en uno de los sistemas funcionales conlleva la pérdida de privilegios en otros, hecho que hace probable una exclusión generalizada.

Esta conceptualización permite abordar fenómenos que la sociología de la educación, en su orientación a la unidad, ha entendido como fenómenos contradictorios. Ciertamente los alumnos que pasan por la escuela aprenden algo para la vida: la pregunta es si esto corresponde a un hecho único o apropiado en forma contextualizada según el sistema en cuestión. La investigación apunta en la segunda dirección, esto es, que lo enseñado es constantemente contextualizado. Por ejemplo, pese a que el profesorado mantenga una actitud crítica ante el rol de la familia y la escuela en la socialización de niños y niñas en roles sexuales específicos, la división entre estudios y carreras masculinas y femeninas permanece válida (Kelly et al. 1982, Spear 1982 y Stanworth 1987 citado en Bonal 1998: 160). En este mismo sentido de abandono de la correspondencia entre educación y sociedad, Lave (1988) indica que en la vida cotidiana no se procede según matemáticas aprendidas en la escuela sino de acuerdo a relaciones de mayor o menor familiaridad. 
Aún en el plano político actualmente esto es reconocido. Se puede asumir que debe tenerse una sociedad democrática pero se reconoce a la vez que en el interior de la sala de clases ésta debe convivir con una limitación de la misma. Según señala Gutmann (1987), los objetivos que están relacionados con la disciplina tienen el mismo valor que los objetivos que buscan fomentar la participación. Esta formulación de una pedagogía que promueve la democracia a través de la educación es también rechazada en el estudio de Latorre (2009), donde se indica que, por sobre contenidos pedagógicos aprendidos en su etapa de formación docente, los profesores utilizan criterios de evaluación derivados de su experiencia escolar como alumnos. Asimismo, el debilitamiento de la relación entre posicionamiento económico y escolaridad forma parte de los lugares comunes de las investigaciones actuales. La movilidad social a través de la educación existe, pero esta es una asociación que cada vez más es reemplazada por otros factores como los años de experiencia laboral, el origen socioeconómico o el sexo (Beyer s/f, Robles 2006).

Todos estos ejemplos nos señalan las dificultades que presenta intentar extender los resultados de las operaciones del sistema educativo a otros sistemas de la sociedad, salvo que nos estemos refiriendo al caso de la exclusión. El aporte teórico y metodológico de Ossandón (2006) sigue una línea alternativa a este planteamiento. La educación no encuentra un correlato en otros sistemas de la sociedad sino formula sus propias dinámicas a partir de una observación diferenciada entre alumnos educables y no educables. A partir de la definición de las bajas expectativas con las cuales los profesores de las escuelas municipales de Chile observan a sus alumnos, Ossandón propone complejizar la diferencia entre inclusión y exclusión en el lado de la inclusión a partir de la distinción entre alumnos educables y no educables. Mientras de los primeros se esperan resultados educativos en el sentido tradicional del término, es decir, selecciones académicamente relevantes, sobre los segundos pesan objetivos orientados al disciplinamiento corporal.

Sobre esta postura llama la atención el abandono de la idea de que las relaciones en la educación repercuten linealmente en otros sistemas. La comunicación educativa produce sus propias distinciones sin que pueda asegurarse que estas sean relevantes (o no sean relevantes) para el resto de la sociedad. Esta decisión le permite Ossandon interrogarse sobre los esquemas de diferencias que utilizan los observadores en la construcción de la realidad. En consecuencia, es ahora posible superar como categoría analítica la distinción entre público y privado -basada en la propiedad económica- por una estrictamente educativa en línea con la primacía descriptiva de la diferenciación funcional. Por último, la utilización de un enfoque orientado a observar las expectativas de los profesores sobre los alumnos tiene enormes posibilidades de enlazarse con la literatura existente sobre la aplicación de la social self-fulfilling prophecy y el Teorema de Thomas (Bonal 1998).

Una segunda propuesta teórica sobre el tema se encuentra en Robles (2006) que también incorpora la reflexión en torno a la diferenciación funcional y sus problemáticas, llegando a calificar a las actuales dinámicas del sistema educativo como cognitivamente cerrado frente a las irritaciones del entorno. Según el autor, la exclusión en el sistema educativo es el resultado de la interferencia de criterios económicos en el nivel del acceso a las organizaciones educativas. Esto queda representado en la imposición del criterio de selección basado en la distinción pagar/no pagar (poseer dinero/no poseer dinero) así como la creciente trivialización de la pedagogía como ciencia irreflexiva respecto a su objeto de interés. Una segunda contribución a esta cuestión se encuentra en la descripción que Robles (2005) propone de las categorías que median la inclusión y la exclusión: inclusión en el sentido tradicional (inclusión en la inclusión), inclusión pero sin redes de soporte informales que aseguren su permanencia (exclusión en la inclusión), exclusión con mecanismos de asociación y resistencia (inclusión en la exclusión) y exclusión entendida en el sentido tradicional del término (exclusión en la exclusión). 
De la misma forma que en Ossandón, las categorías de inclusión no repercuten en otros sistemas funcionales en una forma predecible: así como un alumno educable no necesariamente es un buen trabajador el acceso a una organización educativa no asegura la inclusión en el resto de sistemas de la sociedad. Por otra parte, las nuevas categorías de exclusión en la inclusión e inclusión en la exclusión permiten observar formas de exclusión que subyacen (¿sostienen?) la inclusión educativa permitiendo dar cuenta de características asociadas a la exclusión como observación corporal carente de premisas comunicativas en contextos tradicionalmente vistos como integrados (Luhmann 1998: 194). En el mismo sentido, la categoría de inclusión en la exclusión hace posible describir redes orientadas a la educación en contextos de exclusión, lo cual ha pasado desapercibido para una sociología centrada en las instituciones escolares (Bonal 1998).

La postura de Mascareño, por su parte, comprende un énfasis en la negligencia característica de los sistemas sociales en la diferenciación funcional y sus consecuencias en el sistema educativo $(2000,2005)$. Mediante un recorrido histórico por la diferenciación del sistema educativo frente a la religión y la familia, el sociólogo describe la desigualdad interna, no mediante la postura de la sociología crítica, sino como una consecuencia ineludible de la diferenciación sistémica. Dado que las posiciones en el interior de escuelas y universidades son asignadas mediante la selección social, las diferencias sólo son atribuibles a esta (Luhmann \& Schorr 1996: 57). Por tanto, la desigualdad externa en la forma de correspondencia con otros sistemas se presenta como un producto de la permanencia de resabios de estratificación en la sociedad contemporánea que, dada la primacía de separación funcional, debe acomodarse a esta forma de diferenciación.

Por último, tenemos el aporte de Michailakis \& Reich derivado de su investigación sobre las formas de exclusión que afectan a los limitados intelectuales en Suecia (2009). Partiendo de la definición que Luhmann desarrolla junto a Schorr en relación a que en el sistema educativo se cumplen funciones tanto de educación como de selección, los autores asumen que el cumplimiento casi universal de la primera función no debe hacer omitir problemáticas asociadas a la segunda. Según este planteamiento, en el caso de cierto tipo de alumnos la promoción a un nivel superior de enseñanza no sería consecuencia de la aprobación en los mecanismos de selección (evaluaciones) sino más bien un producto temporal ineludible. De forma paradójica, la exclusión educativa se encuentra asociada en esta propuesta a la no aplicación del código de selección.

La principal utilidad de esta postura es que establece la función de selección sin por ello sobrecargarla como un esquema de causalidad en relación a la inclusión en el resto de sistemas funcionales. Esta argumentación está en línea con la diferenciación entre código y programa puesto que, dado que el código sólo distingue comunicaciones educativas, no puede asegurarse la extensión de la inclusión en otros sistemas funcionales por la vía educativa (Luhmann \& Schorr 1990 y 1996). De esta forma, la no aplicación del código de selección pedagógica es una operación educativa que promueve la exclusión en otros sistemas funcionales, en tanto su aplicación es mucho más débil en provocar la inclusión.

En esta sección hemos descrito cuatro autores que intentan observar la desigualdad en el sistema educativo producto de sus propias operaciones y abandonan el entendimiento de la correlación entre la inclusión educativa y la del resto de sistemas funcionales. Quiero ahora sintetizar las posibilidades de estos lineamientos en el siguiente cuadro a partir de su comparación respecto a los supuestos de sistemas sociales y las posibilidades de relación con otras teorías no sistémicas (Cuadro 1). 
Cuadro 1: Comparación entre investigaciones

\begin{tabular}{|c|c|c|c|c|}
\cline { 2 - 5 } \multicolumn{1}{c|}{} & Ossandón & Robles & Mascareño & Michailakis y Reich \\
\hline Teoría de la diferenciación & + & + & + & + \\
\hline Selectividad pedagógica & \pm & \pm & + & + \\
\hline Diferenciación académica interna & + & + & + & + \\
\hline No correlación educación y sociedad & \pm & + & + & \pm \\
\hline Relaciones fuera de TSS & \pm & - & \pm & - \\
\hline
\end{tabular}

El abandono de las observaciones que asocian a la educación como el medio desde el cual asegurar la igualdad o la desigualdad en la sociedad es reemplazado por la utilización de la teoría de la diferenciación funcional de sistemas autorreferenciales. Sobre este punto existe consenso en las investigaciones en que la escuela no es una instancia socializadora que promueva el orden social ni que asigne posiciones posibles de extender a otros ámbitos de la sociedad sino que asigna posiciones a partir de operaciones internas. De esta forma, los aspectos económicos y políticos juegan un rol secundario únicamente integrado a la codificación sistémica mediante los programas. En diferentes niveles de análisis todos los autores apuntan a esta característica. Mientras Ossandón otorga un mayor valor a las expectativas sobre el educando, Mascareño apunta a la descripción de las formas que toman los resabios de estratificación en un sistema educativo diferenciado y Robles define qué sucede en la selectividad pedagógica en las organizaciones, donde el código pago/no pago es impuesto. Nótese que ninguna de estas posturas es contrapuesta: es perfectamente admisible que elementos de la sociedad estratificada tomen formas propias de la diferenciación funcional a partir de la distinción entre alumnos educables y no educables, así como en la extensión de criterios económicos a la educación.

En lo referente a la selectividad pedagógica existe una mayor matización entre los autores señalados. Si bien todos presuponen la generación de diferencias en forma autorreferencial, en algunos esto se analiza con detalle en tanto otros únicamente dejan implícitas sus posibilidades. En este sentido, Ossandón y Robles dejan pendiente la profundización acerca de la forma en que influyen las expectativas docentes y la exclusión en la inclusión en la selectividad pedagógica. Si, como argumenta Robles (2006), se está en presencia de un sistema educativo dual como consecuencia de la interferencia del medio dinero en las operaciones sistémicas, ¿en qué medida esto queda de manifiesto en la utilización de una selección menos pedagógica, es decir, menos centrada en la evaluación de premisas académicas? Idéntica interrogante es posible realizar en el trabajo de Ossandón pese a que las consecuencias que señala permiten aventurar la primacía de una selección disciplinaria antes que pedagógica sobre los alumnos observados como no educables. En el caso tanto de Mascareño como de Michailakis \& Reich la imposición del código de selección cumple un rol importante como el espacio donde la exclusión educativa se realiza en la sociedad funcionalmente diferenciada.

La diferenciación académica interna entendida como selección pedagógica está presente en todos los autores con pequeñas variaciones. Mientras Ossandón, Michailakis \& Reich denuncian su reemplazo por esquemas de disciplinamiento corporal y promoción automática de cursos inferiores a superiores, respectivamente, Robles y Mascareño dan cuenta de su primacía matizada por la interferencia de otros sistemas en los programas. Todos los autores concuerdan en que en el sistema escolar se diferencia según rendimiento académico, si bien en la exclusión este fenómeno se hace improbable.

Bajo este concepto nos referimos a la incapacidad de asegurar la inclusión por la vía educativa. En este respecto las posiciones teóricas varían ligeramente. Ossandón reconoce 
la preponderancia de una distinción educativa, sin mencionar explícitamente si esta encuentra un correlato en el resto de sistemas sociales. Robles parte de la premisa de que criterios económicos intervienen en los programas educativos, si bien esto no lo lleva a afirmar que la educación es la piedra angular en la superación o reproducción de la desigualdad, al contrario, rechaza las teorías que comprenden la pobreza como un círculo acumulativo. Mascareño, por su parte, describe la imposibilidad de la igualdad por la vía educativa ante la primacía de la diferenciación funcional que socava la integración entre inclusión educativa y en el resto de sistemas sociales. Finalmente, pese a que Michailakis \& Reich no describen la correlación entre exclusión educativa y sociedad, sí establecen que la inclusión educativa es una garantía para el desarrollo, especialmente económico.

Sobre las posibilidades de relación fuera de la teoría de sistemas la investigación de Ossandón parece ser relacionable con los estudios sobre expectativas en el ámbito docente. La literatura sobre esto es extensa y proviene principalmente desde el estudio de Rosenthal \& Jacobson sobre el efecto Pigmalión (1968). Por su parte, las categorías propuestas por Robles en torno a la inclusión y la exclusión pueden verse complementadas con estudios realizados por las teorías del capital humano. De hecho, en el estudio citado (2005) el autor fundamenta su descripción de un sistema dual de educación a partir de estadísticas relativas al quintil de ingreso de ingreso familiar. En tercer lugar, el análisis de Mascareño sobre las expectativas puestas en la educación introduce la posibilidad de combinar la revisión historiográfica con este objetivo (2000). Por último, la posición de Michailakis y Reich parece tener menores posibilidades de asociación con ideas fuera de la teoría de sistemas. Esto puede deberse tanto a su orientación totalmente sistémica como a que la investigación sociológica no se ha desarrollado tan ampliamente en torno al código de selección como sobre la comunicación educativa (Luhmann \& Schorr 1990: 58).

\section{Reflexiones finales.}

Las alternativas mencionadas están lejos de ser las únicas provenientes desde la teoría de sistemas. No pretenden ser una solución definitiva a los problemas tradicionales de la sociología de la educación sino ofrecer una alternativa a las descripciones que parten de la premisa de la extensión entre la inclusión educativa y la inclusión en el resto de la sociedad. Consideramos que a través de esta selección se hace posible observar características específicas de los sistemas educativos que, al subordinar sus operaciones a las lógicas de otros ámbitos de la sociedad, permanecían ocultos. En este sentido hacemos nuestra la crítica de Bernstein a estos tipos de análisis sociológico. "Muchos de los análisis (...) asumen el auténtico discurso que someten a su análisis. En particular, estas teorías consideran el discurso pedagógico como medio para otras voces: clase social, género, raza. Los discursos de educación se analizan por su poder para reproducir relaciones dominante/dominado, externas al discurso que, sin embargo, penetran en las relaciones sociales, los medios de transmisión y la evaluación del discurso pedagógico" (Bernstein 1993: 167). Sin problema podríamos agregar a esta lista las relaciones económicas, orientaciones democráticas, disposiciones de conducta y así sucesivamente.

Dicho esto, ¿qué es lo que puede aportar la teoría de sistemas frente al abordaje de la desigualdad en la educación? Una definición rigurosa no es la última de las respuestas. Como señala Luhmann (1991), es necesario reformular el problema con la claridad suficiente para mejorar las orientaciones del sistema respecto a su entorno, para de esta forma prever las repercusiones en la sociedad y los ámbitos del problema más transparentes y con mayores posibilidades de control. En este sentido, sería un error asumir que esta es una perspectiva decantada de una vez y para siempre. En particular, el presente trabajo ha intentado mostrar la presencia de un discurso común en las teorías sociológicas funcionalistas, críticas y del capital humano en torno a las posibilidades de inclusión de la educación. La opción de la teoría de sistemas parece favorecer en este plano la señalización de la educación como un orden autorreferencial emergente cuyas 
consecuencias distan de ser predecibles según las operaciones de otros sistemas funcionales. Con este fin, hicimos una revisión de propuestas teóricas que abordan, respetando la premisa indicada, la problemática de la desigualdad en la educación desde la distinción entre inclusión y exclusión, procurando indicar que es posible adecuar la teoría sociológica a una sociedad en la cual la inclusión dista de estar asegurada.

No es extraño que la sociología de la educación aspire a definir el objeto de sus preocupaciones con cualidades extensibles al resto de la sociedad. Idéntica operación es identificable tanto para el sistema financiero y su observación de la economía como para una pareja de enamorados que aseguran que el amor o desamor es el motor del mundo. Sin embargo, bajo la utilización de nuevos esquemas de diferencias la sociología tiene la oportunidad de dejar de ser observación de la unidad social representada en la educación para decantar en observación de la diferencia, de lo que distingue al sistema funcionalmente diferenciado de la educación respecto a los demás sistemas y cómo sus irritaciones son procesadas autorreferencialmente en la sociedad. En este sentido, si bien es posible que la educación haya sido central en los tiempos que los clásicos de la disciplina tuvieron que describir, sería un error continuar utilizando su acervo intelectual en una sociedad radicalmente diferente que ha demostrado -con creces- la imposibilidad de la coordinación de la igualdad o la desigualdad a través de la educación. De haber motivado este trabajo a la reflexión en esta dirección el objetivo está más que logrado. RM

\section{Bibliografía}

Althusser, L. (1985). El aparato ideológico del estado escolar como aparato dominante. En A. Gras (Comp.), Sociología de la educación, Textos fundamentales. Madrid: Narcea.

Bernstein, B. (1985). Clasificación y enmarcación del conocimiento educativo. Revista Colombiana de Educación, 15.

Bernstein, B. (1993). La estructura del discurso pedagógico. Madrid: Madrid.

Beyer, H. (s/f). La urgencia de la educación, Centro de estudios públicos. Disponible en www.cid.harvard.edu/archive/ events/chile/ harald_beyer.pdf 01/ 12/2011)

Beyer, H. (2007). Un sistema educativo con mejores y más fuertes instituciones. En Institucionalidad para el Desarrollo: Los Nuevos Desafíos (pp. 75-100). Santiago de Chile: Centro de Estudios Públicos, Expansiva.

Bonal, X. (1998). Sociología de la educación. Una aproximación crítica a las corrientes contemporáneas. Madrid: Paidós.

Bourdieu, P. \& Passeron, J. (1964). Los herederos. Los estudiantes y la cultura. Buenos Aires: Siglo XXI.

Bourdieu, P. \& Passeron, J. (1977). La reproducción. Elementos para una teoría del sistema de enseñanza. Barcelona: Editorial Laia.

Bowles, S. \& Gintis, G. (1976). Schooling in Capitalist America. New York: Basic Books.

Coleman, J. (1966). Equality of educational theory. Washington D.C: Office of Education.

Contreras, D., Bustos, S. \& Sepúlveda, P. (2007). When Schools are the Ones that Choose: the Effect of Screening in Chile. Social Science Quartely, 91(5), 1349-1368. 
Durkheim, E. (1991). Educación y sociología. México D.F.: Colofón.

Gutmann, A. (1987). Educación democrática. Princeton: Princeton U.P.

Lave, J. (1988). Cognition in practice: Mind, Mathematics and Culture in Everyday Life. Cambridge: Cambridge U.P.

Luhmann, N. (1994). Unidad y diferenciación en la sociedad moderna. Acta Sociologica, $12,55-61$.

Luhmann, N. (1998). Complejidad y modernidad. De la unidad a la diferencia. Madrid: Trotta.

Luhmann, N. (2007). La sociedad de la sociedad. México D.F.: Universidad Iberoamericana, Herder.

Luhmann, N. \& Schorr, K. E. (1990). Presupuestos estructurales de una pedagogía reformista: análisis sociológicos de la pedagogía moderna. Revista de educación, 291, 55- 79.

Luhmann, N. \& Schorr, K. E. (1996). Teoría de la sociedad y pedagogía. Madrid: Paidós.

Mascareño, A. (2000). La ironía de la educación en América Latina. Nueva Sociedad, 165, 1- 10.

Mascareño, A. (2005). La imposibilidad de la igualdad por la vía educativa, Informe $N^{\circ} 513$ Educación, Universidad Católica de Valparaiso.

Massé, C. (2007). El sistema de la educación en Luhmann desde una perspectiva crítica, Cinta de Moebio, 30, 296- 308.

Michailakis, D. \& Reich, W. (2009). Dilemmas of Inclusive Education. ALTER - European Journal of Disability Research, 3(1), 24- 44.

MINEDUC, (2008). Chile hacia el 2050: Proyecciones de Población 1990-2020. Departamento de Estudios y Desarrollo. Instituto Nacional de Estadísticas.

Ossandón, J. (2006). 'Objeto pedagógico perdido'. Exclusión en la inclusión educativa. En I. Farías \& J. Ossandón (Eds.), Observando sistemas. Nuevas apropiaciones y usos de la teoría de Nilkas Luhmann (pp. 71-99). Santiago de Chile: RIL editores, Fundación Soles.

Oviedo, D. (2001). Organizaciones escolares como promotoras de teorías implícitas de desarrollo. Proyecciones sobre las carreras laborales de los alumnos. Tesis para optar al grado de magister en investigación social y desarrollo. Universidad de Concepción.

Parsons, T. (1959). The School Class as a Social System: Some of its Functions in American Society. Harvard Educational Review, 29(4), 297- 318.

Qvortrup, L. (2005). Society's Educational System. An introduction To Niklas Luhmann's Pedagogical Theory. Seminar.net, $1(1)$.

Robles, F. (2005). Contramodernidad y desigualdad social: Individualización e individuación, inclusión/ exclusión y construcción de identidad. La necesidad de una sociología de la exclusión. Revista Mad, 12, 27- 57. 
Robles, F. (2006). Los ecos de la vergüenza: pasado y presente de la exclusión social en Chile. Manuscrito.

Rosenthal, R. \& Jacobson, L. (1968). Pygmalion in the classroom: teacher expectation and pupils intellectual development. New York: Holt, Rinehart \& Winston.

\section{Sobre el autor}

Julio Labraña es sociólogo de la Universidad de Concepción, Chile. Actualmente se desempeña como investigador en la Universidad Federico Santa María- Sede Concepción. Sus áreas de especialización son: Teoría de sistemas sociales, educación, desigualdad y metodologías de investigación.

labrana.j07@gmail.com

\section{Contacto}

Universidad Técnica Federico Santa María- Sede Concepción

Alemparte 943 - Hualpén

Chile

Recibido: Diciembre 2011

Aceptado: Abril 2012 